\title{
The Return of Bedside Rounds
}

\author{
Jed Gonzalo, MD \\ Beth Israel Deaconess Medical Center, Boston, MA, USA.
}

$\mathrm{J}$ Gen Intern Med 26(2):114

DOI: $10.1007 / \mathrm{s} 11606-010-1563-\mathrm{y}$

(c) Society of General Internal Medicine 2010

Authors Reply: We thank Drs. Peltran and Wright for their thoughtful comments and review of our article. We acknowledge that it can be difficult to make direct comparisons between studies on bedside rounds, as the definition of variables and time periods are different. However, critical review of available data does suggest the frequency of bedside rounding encounters and time spent at the bedside have decreased.

In their 1964 article, Reichsman et al. reported the total number of patient encounters during which the attending and house staff saw the patient was $75 \%{ }^{1}$ The $61 \%$ value reported by Crumlish et al. in 2009 does not refer to the number of patient encounters, but rather to rounding sessions that included "at least one" bedside interaction-the study was not designed to answer the question of frequency. Using their average census size of 12 , the frequency of bedside encounters could have been as low as 5\%. ${ }^{2}$ Applying our definition of "bedside rounds," which includes presentations at the bedside, a proportion of the $75 \%$ reported by Reichsman et al. were "bedside rounds," greater than our frequency of $<1 \%$ prior to the intervention. These numbers suggest a decrease in frequency of both bedside encounters and "bedside rounds."

Payson et al. in 1965 reported the proportion of time the attending spent at the bedside per rounding session was $19.2 \%$, but they also report percentage of time spent at the bedside during "resident rounds" (56.5\%) and "special attending rounds" $(11.5 \%)$, both of which comprised a significant portion of their rounding sessions. ${ }^{3}$ The study did not seek to

answer total rounding time, but using their reported " 1 hour" for resident work rounds, "2 hours" for attending rounds, and " 2 hours" for special attending rounds as denominators, the average time at least two physicians spent at the bedside per rounding session would have been 70 minutes. In the encounters analyzed by Crumlish et al., the average was 17 minutes.

The more important question, however, is if we believe the current time spent at the bedside is sufficient to accomplish the educational and patient care goals for the residents and patients on our medicine services. As stated in our discussion, eloquently written in numerous editorials, reported by patients and house staff, and alluded to by Drs. Peltran and Wright, bedside teaching and learning needs to be "promoted."4,5 Further efforts should be encouraged to this end.

Corresponding Author: Jed Gonzalo, MD; Beth Israel Deaconess Medical Center, 330 Brookline Avenue, Boston, MA 02115, USA (e-mail: jgonzalo@bidmc.harvard.edu).

\section{REFERENCES}

1. Reichsman F, Browning FE, Hinshaw JR. Observations of undergraduate clinical teaching in action. J Med Educ. 1964;39:147-63.

2. Crumlish CM, Yialamas MA, McMahon GT. Quantification of bedside teaching by an academic hospitalist group. J Hosp Med. 2009;4(5):304-7.

3. Payson HE, Barchas JD. A time study of medical teaching rounds. N Engl J Med. 1965;273(27):1468-71.

4. Verghese A. Culture shock-patient as icon, icon as patient. N Engl J Med. 2008;359(26):2748-51.

5. Alpert JS. Some thoughts on bedside teaching. Am J Med. 2009;122 (3):203-4. 